\title{
Inhomogeneity Effect on Solitary Structures in a Magnetized Warm Plasma: Ionization Versus Recombination
}

\author{
Jyoti $^{1}$, Hitendra K. Malik ${ }^{*}, 1$ and R. P. Dahiya ${ }^{2}$ \\ ${ }^{I}$ Plasma Waves and Particle Acceleration Laboratory, Department of Physics, Indian Institute of Technology Delhi, \\ New Delhi - 110 016, India \\ ${ }^{2}$ Centre for Energy Studies, Indian Institute of Technology Delhi, New Delhi - 110 016, India
}

\begin{abstract}
In a magnetized inhomogeneous warm plasma having ionization/recombination, usual version of the KdV equation is found to be modified by two additional terms arising due to the inclusion of ionization/recombination and the density gradient in the plasma. The density gradient in the plasma shows its dependence on the ionization/recombination rate, ion-to-electron temperature ratio, obliqueness of the magnetic field and drift velocity of the ions. In the considered plasma, only the compressive solitary structures are found to propagate corresponding to two different types of modes. A significant effect of magnetic field and ion temperature is found on these structures in both the cases of ionization and recombination, though these structures show weak dependence on the charge of the ions. Interestingly the solitons with prominent tailing structures are evolved in the plasma under the effect of stronger density gradient and higher ionization or recombination. The tailing structure with bigger size evolves in the plasma in the case of only recombination, suggesting that the exchange/transfer of energy from the main soliton to its tail is on a greater scale in the case of recombination than the case of ionization. There exists a critical value of the ionization rate at which the tailing structure associated with only the fast (not slow) solitary structure is vanished. Similarly, the tailing structure associated with only the slow (not fast) solitary structure is disappeared at a critical value of recombination rate.
\end{abstract}

Keywords: Ionization, magnetized plasma, recombination, solitary wave.

\section{INTRODUCTION}

Solitary waves are special kind of waves which neither grow nor dissipate and preserve their shape during the propagation despite the nonlinearity and dispersion of the medium. If a solitary wave during its collision with another solitary wave retains its shape, it is called a soliton (because of the particle like nature). The soliton structure can travel up to a longer distance without losing its energy. Owing to this property, the solitons are very useful in the transportation of energy from one place to the other in laboratory, astrophysical and space related plasmas. Electromagnetic solitary structures have been used in the communication as well.

The first analysis with regard to the solitary wave propagation in a plasma was given by Washimi and Taniuti [1], where they had derived the Korteweg-deVries (KdV) equation and showed that this equation governs the behavior of solitary wave. An extensive review on the ion-acoustic waves and solitons has been made in different situations including both homogeneous and inhomogeneous plasmas [2-42]. Kuehl and Imen [11] have investigated the characteristics of an ion-acoustic soliton in an inhomogeneous plasma taking into account the full set of

*Address correspondence to this author at the Plasma Waves and Particle Acceleration Laboratory, Department of Physics, Indian Institute of Technology Delhi, New Delhi - 110 016, India;

Tel: +91-11-2659-1303, 2051-4221; Fax: +91-11-2658-1114, 2037, 2277;

E-mails: hkmalik@physics.iitd.ac.in, hkmalik@hotmail.com fluid equations. Nejoh [12] has examined the contribution of finite ion temperature to the soliton propagation characteristics in collisionless relativistic plasma. His theory was extended by Singh and Dahiya [13] for an inhomogeneous plasma, where they had obtained the KdV equation for one-dimensional soliton using Reductive Perturbation Technique (RPT) along with appropriate coordinate transformation. The oblique propagation of solitons has been studied by Aziz and Stroth [14] in an inhomogeneous plasma, where the effect of ion temperature was examined on the soliton propagation characteristics. On the other hand, Nakamura [15-17], Nishida [7, 8, 10] and Lonngren [18-22, 39-41] groups have conducted several experimental studies on solitons in different types of plasmas. In addition to the experimental and theoretical studies, researchers have also investigated the propagation of solitary waves under the effect of magnetic field [23, 25, 28, 32], where they observed different behavior of the solitary waves.

Although an extensive work has been accomplished on the solitary waves, the inhomogeneity has been neglected in most of the investigations, which may arise in the plasmas due to the density gradient, temperature or the magnetic field. In addition, low current discharges may lead to the generation of charged particles in plasmas by the process of ionization of neutral particles that play an important role in the plasma dynamics. If we look at theoretical approaches, we find that mainly two types of models (thermal ionization model and beam ionization model) are appropriate for studying the waves in such plasmas. Thermal ionization 
model assumes the ionization to take place due to thermal electrons and this is applicable to low pressure discharges. The beam ionization model is appropriate to magnetic multidipole devices. Here, the ionization is considered to be carried out by a small group of fast electrons that have energies much larger than that of the bulk plasma electrons and the density of such fast electrons is taken to be negligibly small compared to the total electron density.

Considering weak discharges, recently we have investigated the solitary wave evolution in a magnetized inhomogeneous plasma under the effect of a constant ionization rate [34]. For the sake of simplification of calculations, however, in [34] we had neglected the finite temperature of the ions and also the recombination. Not only this, the evolution of density gradient due to the ionization and initial drift of the ions was not discussed in the equilibrium/unperturbed state of the plasma. Therefore, in the present article, we consider all these points and also include the recombination. Through the present cumbersome calculations, we derive relevant modified $\mathrm{KdV}$ equations for both the cases of the ionization and recombination (inverse of the ionization). Then we make a comparison study of the solitary wave characteristics in both these situations. Our calculations reveal that there exist two types of compressive solitons with which a tailing like structure is associated. This tailing structure grows prominently under the effect of recombination in comparison with the case of ionization.

\section{BASIC FLUID EQUATIONS}

We consider an inhomogeneous plasma consisting of ions and electrons under the effect of an external magnetic field $\vec{B}$ applied in the $z$-direction. The wave propagation is taken to be oblique at an angle $\theta$ with the direction of magnetic field in the $(\mathrm{x}, \mathrm{z})$ plane. It means the angle $\theta$ is the angle between the directions of magnetic field and wave propagation. The plasma response to the oscillations in the presence of constant ionization is governed by the equations $[34,47,48]$

$$
\begin{aligned}
& \frac{\partial n_{p}}{\partial t}+\left(\hat{x} \frac{\partial}{\partial x}+\hat{z} \frac{\partial}{\partial z}\right) \cdot\left[n_{p}\left(v_{x} \hat{x}+v_{y} \hat{y}+v_{z} \hat{z}\right)\right]-\gamma_{p}=0, \\
& m_{p} n_{p} \frac{\partial\left(v_{x} \hat{x}+v_{y} \hat{y}+v_{z} \hat{z}\right)}{\partial t} \\
& +m_{p} n_{p}\left[\left(v_{x} \hat{x}+v_{y} \hat{y}+v_{z} \hat{z}\right) \cdot\left(\hat{x} \frac{\partial}{\partial x}+\hat{z} \frac{\partial}{\partial z}\right)\right]\left(v_{x} \hat{x}+v_{y} \hat{y}+v_{z} \hat{z}\right), \\
& +n_{p} Z_{p} e\left(\hat{x} \frac{\partial}{\partial x}+\hat{z} \frac{\partial}{\partial z}\right) \varphi-n_{p} Z_{p} e\left[\left(v_{x} \hat{x}+v_{y} \hat{y}+v_{z} \hat{z}\right) \times \vec{B}\right] \\
& +C_{p} T_{p}\left(\hat{x} \frac{\partial}{\partial x}+\hat{z} \frac{\partial}{\partial z}\right) n_{p}+m_{p} \gamma_{p}\left(v_{x} \hat{x}+v_{y} \hat{y}+v_{z} \hat{z}\right)=0 \\
& n_{e}-n_{e 0} \exp \left(\frac{e \varphi}{T_{e}}\right)=0, \\
& \left(\frac{\partial^{2}}{\partial x^{2}}+\frac{\partial^{2}}{\partial z^{2}}\right) \varphi-\left(n_{e}-Z_{p} n_{p}\right) e / \varepsilon_{0}=0 .
\end{aligned}
$$

Eq. (1) is the continuity equation and Eq. (2) is the equation of motion of the ion fluid. The electrons are assumed to follow the Boltzmann distribution that yields Eq. (3) for the electron fluid. Finally, Poisson's equation [Eq. (4)] is used that connects the electric field and charges during the oscillation of ion and electron fluids. In Eqs. (1) - (4), $n_{p}$ and $n_{e}$ are the concentrations of ions and electrons, respectively, $\varphi$ is the electric potential, $Z_{p} e$ is the amount of charge associated with a single ion, $v_{x}, v_{y}$ and $v_{z}$ are the velocity components of the ion fluid in the $\mathrm{x}-, \mathrm{y}$ - and z-directions, $\gamma_{p}$ is the ionization rate, $m_{p}$ is the ion mass, $C_{p}$ is the specific heat ratio at constant pressure, $T_{p}\left(T_{e}\right)$ is the ion (electron) temperature in $\mathrm{eV}$, and $\vec{B}\left(\equiv B_{0} \hat{z}\right)$ is the magnetic field.

The basic Eqs. (1) - (4) are written in the dimensionless form. For this, the densities are normalized by the unperturbed plasma density $n_{p 0}$, velocities by the ion acoustic speed $C_{s}\left(\equiv \sqrt{\frac{T_{e}}{m_{p}}}\right)$, electric potential by $\frac{T_{e}}{e}$, distance by $\frac{C_{s}}{\omega_{p i}}$; time by $\omega_{p i}^{-1}$ and $\gamma_{p}$ by $\omega_{p i} n_{p 0}$, where $\omega_{p i}\left(\equiv \sqrt{\frac{n_{p 0} Z_{p}^{2} e^{2}}{\varepsilon_{0} m_{p}}}\right)$ is the ion plasma frequency. The unperturbed density with which we have normalized the ion and the electron densities is the density at some reference point, say $\mathrm{x}=\mathrm{z}=0$. We can write this as $n_{p 00}\left(\equiv n_{p 0}\right)$ for the easy understanding of the readers. So the normalized densities are $n_{p}^{\prime}=n_{p} / n_{p 00}$ and $n_{e}^{\prime}=n_{e} / n_{p 00}$ together with their equilibrium (unperturbed) values as $n_{p 0}^{\prime}=n_{p 0} / n_{p 00}$ and $n_{e 0}^{\prime}=n_{e 0} / n_{p 00}$, where $n_{p 0}$ and $n_{e 0}$ are the unperturbed densities at the point other than the reference point. Similarly, the normalized velocities are $v_{x}^{\prime}, v_{y}^{\prime}$ and $v_{z}^{\prime}$ together with their equilibrium values as $v_{x 0}^{\prime}, v_{y 0}^{\prime}$ and $v_{z 0}^{\prime}$, respectively. The normalized potential is $\varphi^{\prime}$ together with its equilibrium value as $\varphi_{0}^{\prime}$, normalized ionization rate is $\gamma_{p}^{\prime}$, normalized time is $t^{\prime}$ and normalized space coordinates are $x^{\prime}$ and $z^{\prime}$. For the sake of simplicity, we henceforth remove the prime from these quantities. Finally, Eqs. (1) - (4) take the following form after normalization:

$$
\begin{aligned}
& \frac{\partial n_{p}}{\partial t}+\frac{\partial\left(n_{p} v_{x}\right)}{\partial x}+\frac{\partial\left(n_{p} v_{z}\right)}{\partial z}-\gamma_{p}=0, \\
& n_{p} \frac{\partial v_{x}}{\partial t}+n_{p} v_{x} \frac{\partial v_{x}}{\partial x}+n_{p} v_{z} \frac{\partial v_{x}}{\partial z}+n_{p} Z_{p} \frac{\partial \varphi}{\partial x}- \\
& n_{p} Z_{p} A v_{y}+2 \sigma_{p} \frac{\partial n_{p}}{\partial x}+\gamma_{p} v_{x}=0 \\
& n_{p} \frac{\partial v_{y}}{\partial t}+n_{p} v_{x} \frac{\partial v_{y}}{\partial x}+n_{p} v_{z} \frac{\partial v_{y}}{\partial z}+ \\
& n_{p} Z_{p} A v_{x}+\gamma_{p} v_{y}=0
\end{aligned}
$$


$n_{p} \frac{\partial v_{z}}{\partial t}+n_{p} v_{x} \frac{\partial v_{z}}{\partial x}+n_{p} v_{z} \frac{\partial v_{z}}{\partial z}+$

$n_{p} Z_{p} \frac{\partial \varphi}{\partial z}+2 \sigma_{p} \frac{\partial n_{p}}{\partial z}+\gamma_{p} v_{z}=0$

$n_{e}-n_{e 0} \exp (\varphi)=0$,

$Z_{p}^{2}\left(\frac{\partial^{2} \varphi}{\partial x^{2}}+\frac{\partial^{2} \varphi}{\partial z^{2}}\right)-n_{e}+Z_{p} n_{p}=0$

where $\sigma_{p}=\frac{T_{p}}{T_{e}}$ is the ion to electron temperature ratio, $A \equiv \sqrt{\frac{\varepsilon_{0} B_{0}^{2}}{n_{p 0} Z_{p}^{2} m_{p}}}$ is the ion cyclotron frequency to ion plasma frequency ratio, and $C_{p}$ has been taken as 2 in view of ions' compression in the $(\mathrm{x}, \mathrm{z})$ plane, i.e. due to number of degrees of freedom as 2 .

Taking $\lambda_{0}$ as the phase velocity of the wave and following $[23,33,34]$, we introduce the following stretched coordinates and the expansion of physical quantities

$\xi=\varepsilon^{1 / 2}\left(\frac{\hat{k} \cdot \vec{r}}{\lambda_{0}}-t\right)=\varepsilon^{1 / 2}\left(\frac{x \sin \theta+z \cos \theta}{\lambda_{0}}-t\right)$,

$\tau=\varepsilon^{3 / 2}(\hat{k} \cdot \vec{r})=\varepsilon^{3 / 2}(x \sin \theta+z \cos \theta)$.

$f=f_{0}(x, z)+\varepsilon^{3 / 2} f_{1}(x, z, t)+\varepsilon^{2} f_{2}(x, z, t)+\ldots ., f \equiv v_{x}, v_{y}$,

$d=d_{0}(x, z)+\varepsilon d_{1}(x, z, t)+\varepsilon^{2} d_{2}(x, z, t)+\ldots ., d \equiv n_{p}, n_{e}, \varphi, v_{z}$,

$\gamma_{p}=\varepsilon^{3 / 2} \gamma_{p 1}$.

We assume the ionization to take place at a constant rate along with $\gamma_{p 1}$ as the rate of ionization per unit volume. Owing to its constant magnitude and for the sake of simplicity, we take $\gamma_{p 1}=\gamma_{p}$ in the forthcoming sections. Based on the use of Eqs. (11) - (15) in Eqs. (5) - (10), we obtain the following zeroth order equations:

$$
\begin{aligned}
& \sin \theta \frac{\partial\left(n_{p 0} v_{x 0}\right)}{\partial \tau}+\cos \theta \frac{\partial\left(n_{p 0} v_{z 0}\right)}{\partial \tau}-\gamma_{p}=0, \\
& \left(v_{x 0} \sin \theta+v_{z 0} \cos \theta\right) \frac{\partial v_{x 0}}{\partial \tau}+Z_{p} \sin \theta \frac{\partial \varphi_{0}}{\partial \tau}+ \\
& \frac{2 \sigma_{p} \sin \theta}{n_{p 0}} \frac{\partial n_{p o}}{\partial \tau}+\frac{\gamma_{p} v_{x 0}}{n_{p 0}}=0 \\
& \left(v_{x 0} \sin \theta+v_{z 0} \cos \theta\right) \frac{\partial v_{z 0}}{\partial \tau}+Z_{p} \cos \theta \frac{\partial \varphi_{0}}{\partial \tau}+ \\
& \frac{2 \sigma_{p} \sin \theta}{n_{p 0}} \frac{\partial n_{p o}}{\partial \tau}+\frac{\gamma_{p} v_{z 0}}{n_{p 0}}=0 \\
& n_{e 0}-Z_{p} n_{p 0}=0, \\
& n_{e 0}=n_{e 0} \exp \left(\varphi_{0}\right) .
\end{aligned}
$$

The zeroth - order Eqs. (16) - (20) are used in the higher order equations. However, we can also draw the following information regarding the plasma in its equilibrium/unperturbed state based on which the phase velocity relation and the $\mathrm{KdV}$ equation are obtained. From Eq. (20) we can write $\varphi_{0}=0$ which implies that $\frac{\partial \varphi_{0}}{\partial \tau}=0$. This is consistent with the observation of other investigators $[30,47,48,53,54]$. Using this in Eqs. (17) and (18), we get

$$
\frac{\partial n_{p o}}{\partial \tau}=\frac{2 \gamma_{p}\left(v_{x 0} \sin \theta+v_{z 0} \cos \theta\right)}{\left(v_{x 0} \sin \theta+v_{z 0} \cos \theta\right)^{2}-2 \sigma_{p}} \text {. }
$$

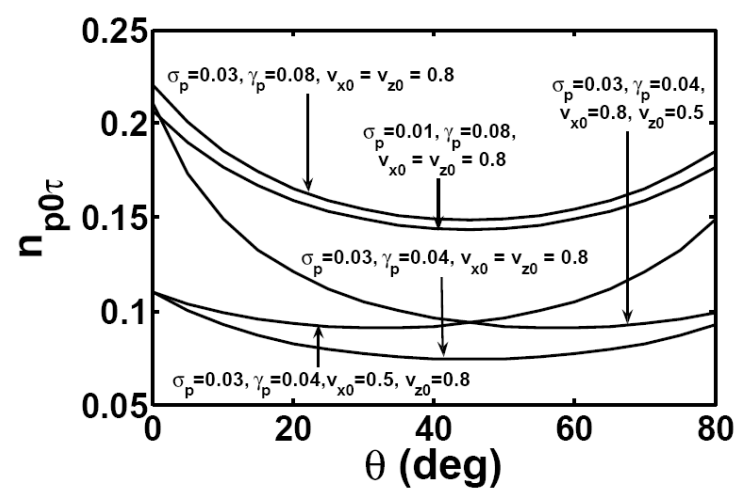

Fig. (1). Variation of density gradient with angle of obliqueness $\theta$ for different values of ion to electron temperature ratio $\sigma_{p}$ and the ionization rate $\gamma_{\mathrm{p}}$. Here, we show the cases of $v_{x 0}=v_{z 0}$ and $v_{x 0} \neq v_{z 0}$.

From this relation, we can analyze the equilibrium/unperturbed state of the plasma by looking at the dependence of the density gradient on the obliqueness of magnetic field $(\theta)$, ionization rate $(\gamma)_{p}$, ion to electron temperature ratio $\left(\sigma_{p}\right)$ and the drift velocity of the ions $\left(v_{x 0}\right.$ and $\left.v_{z 0}\right)$. For better understanding, in Fig. (1), we have shown the variation of $n_{p o \tau}\left(\equiv \frac{\partial n_{p 0}}{\partial \tau}\right)$ with $\theta$ for different values of $\sigma_{\mathrm{p}}, \gamma_{\mathrm{p}}, v_{x 0}$ and $v_{z 0}$. From the figure this is clear that the higher ionization rate and ion temperature lead to higher density gradient in the plasma. The density gradient attains a minimum value, when the angle $\theta$ is $45^{\circ}$ in the case of equal drift velocity components $v_{x 0}$ and $v_{z 0}$. This is obvious, as the Lorentz force introduces inhomogeneity if the magnetic field is applied at angles other than $45^{\circ}$ due to its unequal amounts in the $x$ - and $z$-directions. However, for unequal drift velocities of $v_{x 0}$ and $v_{z 0}$, this angle is different from $45^{\circ}$ and is as per Eq. (21).

The first order equations are obtained as

$\left(\frac{\sin \theta v_{x 0}}{\lambda_{0}}+\frac{\cos \theta v_{z 0}}{\lambda_{0}}-1\right) \frac{\partial n_{p 1}}{\partial \xi}+\frac{\cos \theta}{\lambda_{0}} n_{p 0} \frac{\partial v_{z 1}}{\partial \xi}=0$, 


$$
\begin{aligned}
& \left(\frac{\sin \theta v_{x 0}}{\lambda_{0}}+\frac{\cos \theta v_{z 0}}{\lambda_{0}}-1\right) n_{p 0} \frac{\partial v_{z 1}}{\partial \xi}+\frac{Z_{p} \cos \theta}{\lambda_{0}} n_{p 0} \frac{\partial \varphi_{1}}{\partial \xi} \\
& +\frac{2 \sigma_{p} \cos \theta}{\lambda_{0}} \frac{\partial n_{p 1}}{\partial \xi}+2 \sigma_{p} \cos \theta \frac{\partial n_{p 0}}{\partial \tau} \\
& +\left(v_{x 0} \sin \theta+v_{z 0} \cos \theta\right) n_{p 0} \frac{\partial v_{z 0}}{\partial \tau} \\
& +Z_{p} \cos \theta n_{p 0} \frac{\partial \varphi_{0}}{\partial \tau}+\gamma_{p} v_{z 0}=0 \\
& \frac{\sin \theta}{\lambda_{0}} Z_{p} n_{p 0} \frac{\partial \varphi_{1}}{\partial \xi}-Z_{p}\left(\frac{\varepsilon_{0}}{n_{p 00} Z_{p}^{2} m_{p}}\right)^{1 / 2} B_{0} n_{p 0} v_{y 1} \\
& +\frac{2 \sigma_{p} \sin \theta}{\lambda_{0}} \frac{\partial n_{p 1}}{\partial \xi}+2 \sigma_{p} \sin \theta \frac{\partial n_{p 0}}{\partial \tau} \\
& +\left(\sin \theta v_{x 0}+\cos \theta v_{z 0}\right) n_{p 0} \frac{\partial v_{x 0}}{\partial \tau} \\
& +Z_{p} n_{p 0} \sin \theta \frac{\partial \varphi_{0}}{\partial \tau}+\gamma_{p} v_{x 0}=0 \\
& Z_{p}\left(\frac{\varepsilon_{01}-Z_{p} n_{p 1}=0}{n_{p 00} Z_{p}^{2} m_{p}}\right)^{1 / 2} B_{0} v_{x 1}+\gamma_{p} v_{y 1}=0 \\
& n_{e 1}=n_{e 0} \varphi_{1} \exp \left(\varphi_{0}\right),
\end{aligned}
$$

In order to obtain the phase velocity relation, we make use of Eqs. (22) - (27). In the present case of inhomogeneous plasma, the unperturbed (zeroth order) quantities are functions of only $\tau$. Hence $\frac{\partial f_{0}}{\partial \xi}=0$, where $f \equiv n_{p 0}, n_{e 0}, v_{x 0}, v_{y 0}, v_{z 0}, \varphi_{0}$. Now first order equations are integrated w.r.t. $\xi$ under the boundary conditions that $n_{p 1}, n_{e 1}, v_{x 1}, v_{y 1}, v_{z 1}$ and $\varphi_{1} \rightarrow 0$ as $\xi \rightarrow \infty$ in order to obtain the relations between $n_{p 1}, n_{e 1}, v_{x 1}, v_{z 1}$ and $\varphi_{1}$ [46]. Since zeroth order quantities are self consistently determined by Eqs. (16) - (20), we use these equations in first order equations before their integration. Therefore, the integration of Eqs. (22) - (23) yields

$$
\begin{aligned}
& \left(\frac{v_{x 0} \sin \theta}{\lambda_{0}}+\frac{v_{z 0} \cos \theta}{\lambda_{0}}-1\right) n_{p 1}=\frac{-\cos \theta n_{p 0}}{\lambda_{0}} v_{z 1}, \\
& \left(\frac{v_{x 0} \sin \theta}{\lambda_{0}}+\frac{v_{z 0} \cos \theta}{\lambda_{0}}-1\right) n_{p 0} v_{z 1} \\
& =\frac{-Z_{p} \cos \theta n_{p 0}}{\lambda_{0}} \varphi_{1}-\frac{2 \sigma_{p} \cos \theta}{\lambda_{0}} n_{p 1} .
\end{aligned}
$$

From these relations, we obtain

$$
n_{p 1}=\frac{Z_{p} n_{p 0} \cos ^{2} \theta}{R^{2} \lambda_{0}^{2}-2 \sigma_{p} \cos ^{2} \theta} \varphi_{1} .
$$

Also, Eq. (28) yields

$n_{e 1}=n_{e 0} \varphi_{1}$.

Finally, we use Eq. (27), i.e. $n_{e 1}=Z_{p} n_{p 1}$ and put $n_{p 0} Z_{p}=n_{e 0}$ from Eq. (19) in order to obtain the following quadratic equation in $\lambda_{0}$ :

$\lambda_{0}^{2}-2 \lambda_{0} v_{\theta}+v_{\theta}^{2}-\left(Z_{p}+2 \sigma_{p}\right) \cos ^{2} \theta=0$.

Here, $v_{\theta}=v_{x 0} \sin \theta+v_{z 0} \cos \theta$. The phase velocity relation that we obtain after solving the above quadratic equation is as follows:

$$
\lambda_{0}=v_{x 0} \sin \theta+v_{z 0} \cos \theta \pm \cos \theta \sqrt{Z_{p}+2 \sigma_{p}} .
$$

Corresponding to \pm sign in Eq. (33), we get two types of the phase velocity relations. The relation corresponding to plus sign yields the fast mode (say phase velocity $\lambda_{F}$ ), whereas the relation with minus sign corresponds to the slow mode (say phase velocity $\lambda_{s}$ ). For the propagation of these modes, their phase velocities should be positive (as the direction of wave propagation has been fixed as per stretched coordinates). Here, it is obtained that $\lambda_{F}$ always remains positive, but the following condition on the wave propagation angle $\theta$ should be satisfied for the propagation of the slow mode (for $\lambda_{S}$ to be positive)

$\theta>\tan ^{-1}\left(\frac{\sqrt{Z_{p}+2 \sigma_{p}}-v_{z 0}}{v_{x 0}}\right)$.

Eq. (34) shows that there exists a minimum wave propagation angle ( say $\theta_{\min }$ ), below which the slow mode does not propagate in the plasma. The angle $\theta_{\min }$ is given as

$$
\theta_{\min }=\tan ^{-1}\left(\frac{\sqrt{Z_{p}+2 \sigma_{p}}-v_{z 0}}{v_{x 0}}\right) \text {. }
$$

For better understanding of this, we have plotted in Fig. (2) the variation of $\theta_{\min }$ with the temperature ratio $\sigma_{p}$ and the charge $Z_{\mathrm{p}}$ on the ions. Evidently the minimum angle $\theta_{\min }$ attains larger values for the ions having charge $Z_{p}>1$; the same is the case for the plasma having the ions of higher temperature. Since the ion oscillations experience larger force due to magnetic field applied at a larger angle, it is understood that the slow mode is excited in the plasma only in the presence of a stronger magnetic field. Moreover, the slopes of the solid lines in the figure reveal that $\theta_{\min }$ varies slowly with the ion temperature, if the ions carry higher charge $Z_{p}$. On the other hand, the angle $\theta_{\min }$ is found to reduce for the higher values of $v_{x 0}$ and $v_{z 0}$.

\section{MODIFIED KDV (MKDV) EQUATION AND ITS SOLUTION}

In order to derive the relevant $\mathrm{KdV}$ equation, we make use of higher order equations. In this context, the second order equations are obtained as: 


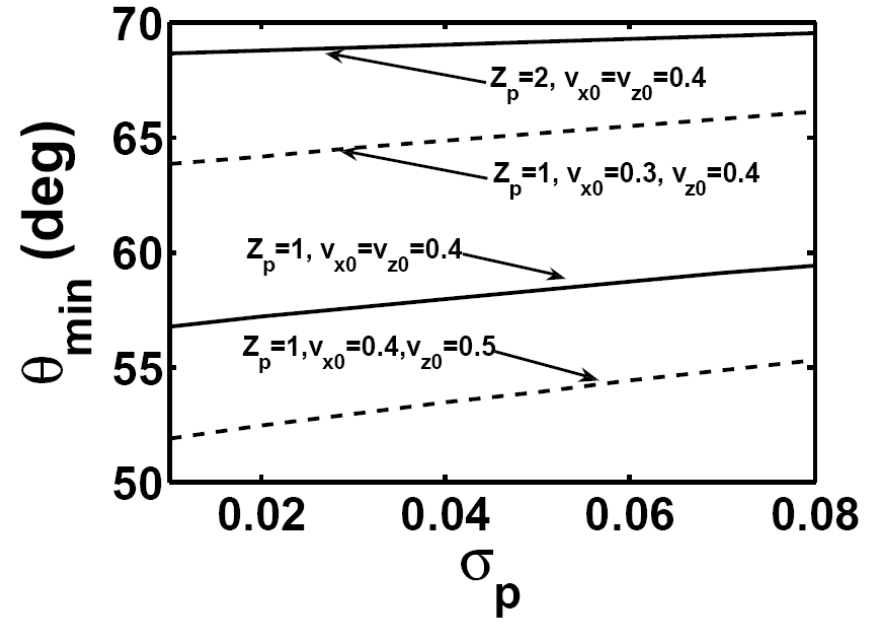

Fig. (2). Variation of $\theta_{\min }$ with $\sigma_{p}$ (ion to electron temperature ratio) for the two cases of $v_{x 0}=v_{z 0}$, and when $v_{x 0} \neq v_{z 0}$ for $\mathrm{Z}_{\mathrm{p}}=1$.

$$
\begin{aligned}
& R n_{p 0} \frac{\partial v_{x 2}}{\partial \xi}+Z_{p} n_{p 0} \sin \theta\left(\frac{1}{\lambda_{0}} \frac{\partial \varphi_{2}}{\partial \xi}+\frac{\partial \varphi_{1}}{\partial \tau}\right) \\
& +2 \sigma_{p} \sin \theta\left(\frac{1}{\lambda_{0}} \frac{\partial n_{p 2}}{\partial \xi}+\frac{\partial n_{p 1}}{\partial \tau}\right) \\
& +v_{z 1} \cos \theta n_{p 0} \frac{\partial v_{x 0}}{\partial \tau}-\frac{2 \sigma_{p} \sin \theta n_{p 1}}{n_{p o}} \frac{\partial n_{p 0}}{\partial \tau}-\frac{\gamma_{p} v_{x 0} n_{p 1}}{n_{p 0}}=0 \\
& R \frac{\partial v_{y 1}}{\partial \xi}+Z_{p}\left(\frac{\varepsilon_{0}}{n_{p 00} m_{p} Z_{p}^{2}}\right)^{1 / 2} B_{0} v_{x 2}+\gamma_{p} v_{y 2}=0 \\
& R \frac{\partial v_{z 2}}{\partial \xi}+\left(\sin \theta v_{x 0}+\cos \theta v_{z 0}\right) \frac{\partial v_{z 1}}{\partial \tau}+Z_{p} \cos \theta\left(\frac{1}{\lambda_{0}} \frac{\partial \varphi_{2}}{\partial \xi}+\frac{\partial \varphi_{1}}{\partial \tau}\right) \\
& +\cos \theta\left(\frac{v_{z 1}}{\lambda_{0}} \frac{\partial v_{z 1}}{\partial \xi}+v_{z 1} \frac{\partial v_{z 0}}{\partial \tau}\right)+2 \sigma_{p} \sin \theta\left(\frac{1}{\lambda_{0}} \frac{\partial n_{p 2}}{\partial \xi}+\frac{\partial n_{p 1}}{\partial \tau}\right) \\
& -\frac{2 \sigma_{p} \cos \theta n_{p 1}}{n_{p 0}^{2}}\left(\frac{1}{\lambda_{0}} \frac{\partial n_{p 1}}{\partial \xi}+\frac{\partial n_{p 0}}{\partial \tau}\right)-\frac{\gamma_{p} v_{z 0} n_{p 1}}{n_{p 0}^{2}}+\frac{\gamma_{p} v_{z 1}}{n_{p 0}}=0
\end{aligned}
$$

where

$$
\begin{aligned}
& R=\frac{\sin \theta v_{x 0}}{\lambda_{0}}+\frac{\cos \theta v_{z 0}}{\lambda_{0}}-1, \\
& n_{e 2}=n_{e 0}\left(\frac{\varphi_{1}^{2}}{2}+\varphi_{2}\right), \\
& n_{e 2}-Z_{p} n_{p 2}-\frac{Z_{p}^{2}}{\lambda_{0}^{2}} \frac{\partial^{2} \varphi_{1}}{\partial \xi^{2}}+\frac{Z_{p}^{2}}{\lambda_{0}} \frac{\partial^{2} \varphi_{0}}{\partial \xi \partial \tau}=0 .
\end{aligned}
$$

In obtaining Eq. (36), we have used first order Eq. (24) in order to eliminate the terms containing $v_{y 1}$. Based on the first and second order equations, finally, we obtain the following modified $\mathrm{KdV}(\mathrm{mKdV})$ equation after making use of the phase velocity relation (33):

$$
\frac{\partial \varphi_{1}}{\partial \tau}+K \varphi_{1} \frac{\partial \varphi_{1}}{\partial \xi}+L \frac{\partial^{3} \varphi_{1}}{\partial \xi^{3}}+M \varphi_{1} \frac{\partial n_{p 0}}{\partial \tau}+N \varphi_{1}=0
$$

This equation is different from the usual $\mathrm{KdV}$ equation by two additional terms arising because of the density gradient and ionization in the plasma. The coefficients $\mathrm{K}, \mathrm{L}$, $\mathrm{M}$, and $\mathrm{N}$ in Eq. (42) are given by

$$
\begin{aligned}
& K=\frac{-Z_{p} \cos \theta\left(R^{2} \lambda_{0}^{2}+\sigma_{p} \cos ^{2} \theta\right)}{\lambda_{0}\left\{\sigma_{p} R \lambda_{0} R_{\lambda}-Z_{p} \cos \theta\left(R^{2} \lambda_{0}^{2}-\sigma_{p} \cos ^{2} \theta\right)\right\}}, \\
& L=\left(\frac{Z_{p}^{2} \cos \theta}{\lambda_{0}^{3} R}+\frac{Z_{p} \lambda_{0} R^{3} \sin ^{2} \theta n_{p 0}}{R_{\lambda} Z_{p}^{2} A^{2} \cos \theta}\right) \\
& {\left[\frac{R R_{\lambda}}{2 Z_{p} \cos \theta n_{p 0}\left(R^{2} \lambda_{0}^{2}-\sigma_{p} \cos ^{2} \theta\right)-2 \sigma_{p} n_{p 0} \lambda_{0} R R_{\lambda}}\right]} \\
& M=\left(\frac{Z_{p} \lambda_{0}^{3} R^{2}}{R_{\lambda}}-\frac{Z_{p} \lambda_{0}^{2} R\left(\begin{array}{c}
v_{x 0} \sin \theta \\
+v_{z 0} \cos \theta
\end{array}\right)\left(1+\cos ^{2} \theta\right)}{R_{\lambda}}\right) \\
& {\left[\frac{R R_{\lambda}}{2 Z_{p} \cos \theta n_{p 0}\left(\begin{array}{l}
R^{2} \lambda_{0}^{2} \\
-\sigma_{p} \cos ^{2} \theta
\end{array}\right)-2 \sigma_{p} n_{p 0} \lambda_{0} R R_{\lambda}}\right]} \\
& N=Q_{1}\left(\frac{-R R_{\lambda}}{2 Z_{p} \cos \theta n_{p 0}\left(R^{2} \lambda_{0}^{2}-\sigma_{p} \cos ^{2} \theta\right)-2 \sigma_{p} n_{p 0} \lambda_{0} R R_{\lambda}}\right),
\end{aligned}
$$

together with

$$
\begin{aligned}
Q_{1}= & \frac{-2 \sigma_{p} Z_{p} \lambda_{0} \cos ^{2} \theta}{R_{\lambda}}-\frac{Z_{p} \lambda_{0} \cos \theta \gamma_{p} v_{z o}}{R_{\lambda}} \\
& +\frac{Z_{p} \lambda_{0}^{2} R \gamma_{p}}{R_{\lambda}}\left(-S \sin \theta \cos \theta-\cos ^{2} \theta\right) \\
S= & \frac{\left(v_{z 0} \sin \theta-v_{x 0} \cos \theta\right)}{\left(v_{x o} \sin \theta+v_{z 0} \cos \theta\right)}, \text { and } R_{\lambda}=\frac{\lambda_{0}^{2} R^{2}-2 \sigma_{p} \cos ^{2} \theta}{\lambda_{0}^{2} R} .
\end{aligned}
$$

The above coefficients are not constant but depend on the zeroth order quantities. Therefore, the normal integration process does not qualify for obtaining its soliton solution. Hence, we introduce the following variable [27]

$\varphi_{1}(\xi, \tau)=\phi(\xi, \tau) b(\tau)$,

where $b(\tau) \equiv \exp \left(-\int{ }^{\tau} M \frac{\partial n_{p o}}{\partial \tau^{\prime}} d \tau^{\prime}\right)$ and $\phi(\xi, \tau)$ are the new variables. With this, the Eq. (42) takes the form

$\frac{\partial \phi}{\partial \tau}+K b \phi \frac{\partial \phi}{\partial \xi}+L \frac{\partial^{3} \phi}{\partial \xi^{3}}+N \phi=0$.

Here $\mathrm{Kb}$ is the coefficient of nonlinearity, $\mathrm{L}$ is the coefficient of dispersion, and the coefficient $\mathrm{N}$ accounts for the effect of ionization. The coefficient $\mathrm{L}$ carries the effect of magnetic field via the term A. It means the dispersive properties of the plasma are modified by the external magnetic field. Since a balance between the effects of 
nonlinearity and dispersion leads to the evolution of solitary wave, the ionization and the magnetic field considered in the present analysis shall modify the propagation characteristics of the solitary waves.

We make use of a transformation $\eta=W_{L}\left(\tau-V_{L} \xi\right)$, where $\mathrm{W}_{\mathrm{L}}$ represents the inverse of the width of solitary wave and $V_{L}$ is the velocity of the new frame of reference moving with the solitary wave with respect to the ion acoustic wave of velocity $\lambda_{0}$. Along with the use of transformation $\eta$, Eq. (44) reads

$W_{L} \frac{\partial \phi}{\partial \eta}-K \phi b W_{L} V_{L} \frac{\partial \phi}{\partial \eta}-L W_{L}^{3} V_{L}^{3} \frac{\partial^{3} \phi}{\partial \eta^{3}}+N \phi=0$.

Here it would be worth mentioning that the parameter $b$ depends only on the coordinate $\tau$. More specifically it shows the variation of unperturbed quantities with space including the density gradient ( $\tau$ being the space like coordinate in Eqs. (11) and (12)). However, the coordinate $\eta=W_{L}\left(\tau-V_{L} \xi\right)$, which encompasses both the space and time like coordinates, is used to find the stationary solution of the $\mathrm{mKdV}$ equation (31). Infact $\eta$ is the coordinate of the frame, which is moving with the soliton and $V_{L}$ is the velocity of the soliton with respect to the frame of reference having coordinates as stretched coordinates. If $\phi$ had been the function of $b$, then it would have been wrong to consider $\varphi=\varphi(\eta)$ for finding the solution of Eq. (44). However, this is appropriate to make use of such transformation as only $\varphi$ is the function of $\eta$ in accordance with $\varphi_{1}(\xi, \tau)=\phi(\xi, \tau) b(\tau)$.

The transformation $b$ is used to club the density gradient term with the nonlinear term and then to transform the modified $\mathrm{KdV}$ equation to an equation suitable for solving using the so called sine-cosine method proposed by Yan [55] and employed by several authors [14 and references therein].

Now following Aziz and Stroth [14], Singh and Malik [27], Das and Sarma [48] and Yan [55], we suppose the following solution of Eq. (45):

$\phi(\eta)=\sum_{j=1}^{s} \tanh ^{j-1}(\eta)\left[B_{j} \operatorname{sech}(\eta)+A_{j} \tanh (\eta)\right]+A_{0}$,

where $\frac{d \omega}{d \eta}=\sin \omega$ and $\omega \Leftrightarrow \eta$ transformations are related through $\sin \omega=\sec h \eta$ and $\cos \omega=\tanh \eta$. The balance of the leading order of the linear terms to that of the nonlinear terms of Eq. (45) determines the degree of the polynomial solution (46) as $\mathrm{s}=2$. Hence, the solution becomes

$\phi(\omega)=B_{1} \sin \omega+B_{2} \sin \omega \cos \omega+A_{1} \cos \omega+A_{2} \cos ^{2} \omega+A_{0}$.

The above expression represents the general solution of Eq. (45) and corresponding to the values of coefficients $A_{0}$, $A_{1}, A_{2}, B_{1}$ and $B_{2}$ different types of solutions would be possible. However, the drawback of this method [14, 24, 26, $27,32-34,55]$ is that it yields more number of equations than the number of variables (coefficients $A_{0}, A_{1}, A_{2}$, etc.). This way $n$ number of solutions may be possible. Hence, to be closer to the correct solution and in view of single soliton solution, we restrict our calculations to the case where $B_{1}=B_{2}=0$. This gives

$\phi(\omega)=A_{0}+A_{1} \cos \omega+A_{2} \cos ^{2} \omega$.

The substitution of the formal solution (48) in Eq. (45) changes the differential equation into a trigonometric polynomial identity of the intermediate variable $\omega$, from which we collect all the terms with the same powers in $\cos \omega, \cos ^{m} \omega, \cos ^{m+1} \omega(\mathrm{m}=0,1,2, \ldots)$ etc., and set their coefficients equal to zero. With this we obtain $W_{L}=\sqrt{\frac{1}{2 L V_{L}^{3}}}$ and hence, $A_{0}=\frac{1}{3 K b V_{L}}, A_{1}=\frac{N}{K b W_{L} V_{L}}$ and $A_{2}=\frac{-1}{3 K b V_{L}}$. Finally, the solution of Eq. (45) is obtained as

$$
\begin{aligned}
& \phi(\omega)=\phi(\xi, \tau)=\frac{1}{3 K b V_{L}} \sec h^{2}\left[W_{L}\left(\tau-V_{L} \xi\right)\right] \\
& +\frac{N}{K b W_{L} V_{L}} \tanh \left[W_{L}\left(\tau-V_{L} \xi\right)\right] .
\end{aligned}
$$

The above solution contains two terms, which can be written in a simpler form, as follows:

$\phi_{\text {Complete }} \equiv \phi_{\text {Main }}\left(\sec h^{2}\right.$ term $)+\phi_{\text {Tail }}(\tanh$ term $)$.

The first term of solution (50) determines the main soliton and the second term shows a tailing like structure associated with the soliton.

\section{RESULTS AND DISCUSSION: CASE OF IONIZATION}

It is evident that the present plasma supports the propagation of solitons that are modified by the presence of tailing like structure. Such solitary structures are possible for both the slow and fast modes, which are named as fast solitary structure $\left(\mathrm{F}_{\mathrm{ss}}\right)$ and slow solitary structure $\left(\mathrm{S}_{\mathrm{ss}}\right)$, respectively. Our numerical results show that these structures corresponding to density $n_{p 1}$ attain hill type shape (the shape as shown in Fig. (3) for potential $\phi_{1}$ ). Hence, the compressive solitary structures are supported by the present plasma. In the forthcoming figures, where $\varphi_{\text {Complete }} \equiv \phi_{\text {Complete }}$, we shall investigate the behavior of these structures under the effect of magnetic field (via A), ionization rate $\left(\gamma_{p}\right)$, charge of ions $\left(\mathrm{Z}_{\mathrm{p}}\right)$, temperature of ions $\left(\right.$ via $\left.\sigma_{p}\right)$ and the density gradient $n_{p 0 \tau}$.

In Fig. (3), we have plotted the complete solitary structures for the fast mode as well as for the slow mode. Here, a significant enhancement in the width is observed at stronger magnetic field, which is in contrast to the investigations made in homogeneous and inhomogeneous magnetized plasmas without consideration of the ionization $[24,33]$. Hence, it is understood that the dispersive property of the plasma is modified oppositely in the plasma, when the 


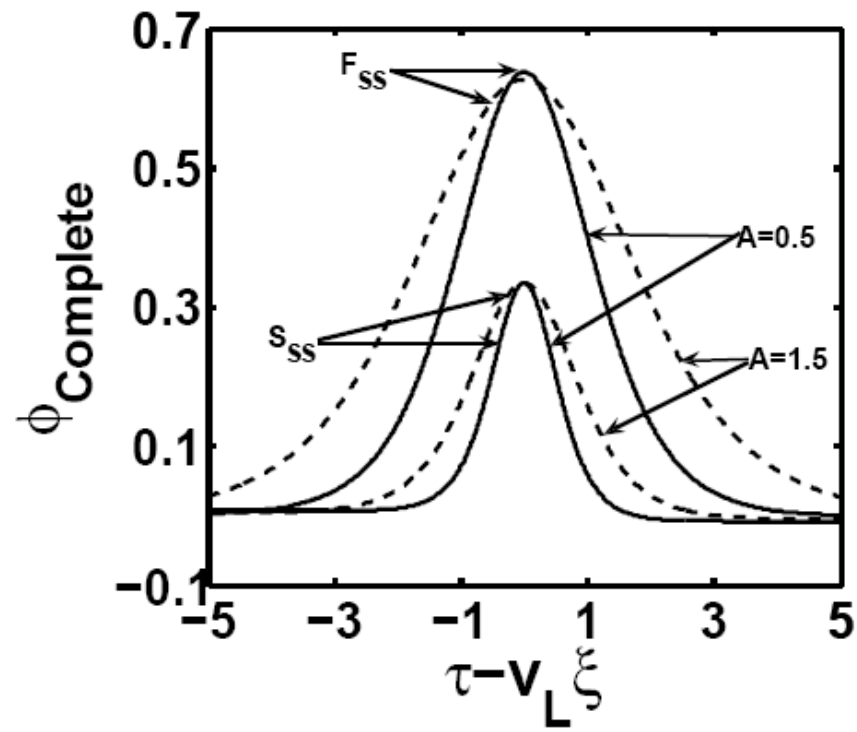

Fig. (3). Fast and slow solitary structures corresponding to $\varphi_{\text {Complete }}$ for two different values of $A \equiv \sqrt{\frac{\varepsilon_{0} B_{0}^{2}}{n_{p 0} Z_{p}^{2} m_{p}}}$, when $\mathrm{n}_{\mathrm{p} 0}=0.9, \gamma_{\mathrm{p}}=0.01$, $\mathrm{Z}_{\mathrm{p}}=1, \theta=50^{\circ}, \sigma_{p}=0.01, v_{x 0}=v_{z 0}=0.08$ for fast mode, and $v_{x 0}=v_{z 0}=0.8$ for slow mode. $\mathrm{F}_{\mathrm{ss}}\left(\mathrm{S}_{\mathrm{ss}}\right)$ shows the fast (slow) solitary structure.

ionization is taken into account; owing to this a wider solitary structure is realized. This type of behavior of the width with magnetic field is also contrary to the observation made by El-Labany and El-Shamy [29] for solitary waves in a hot magnetized dusty plasma and by Mishra et al. [23] in a negative ion containing homogeneous plasma, where they had neglected the ionization. If we examine the solitary profile for the slow mode, the amplitude of this structure is found to be smaller than that of the structure for the fast mode, but consistently there is no effect of the magnetic field on the amplitude. Also, the structure corresponding to the slow mode is narrower. The width of the structure is given by the expression $W_{L}^{-1}=\sqrt{2 L V_{L}^{3}}$, which means the width is real as long as $\mathrm{L}$ is positive. In the present model, we find that $\mathrm{L}$ is positive for both the fast and slow modes, contrary to the case of weakly relativistic plasma [26], in which the width is not real for the fast mode.

In Fig. (4), we have plotted the solitary structures at different rates of the ionization. It is found that due to the presence of ionization the structures get modified drastically. We observe the identical behavior of both the fast and slow solitary structures for the different ionization rates, and their amplitudes are enhanced for the higher ionization in the plasma. It is a point of observation that in the presence of higher ionization the tailing structure takes the prominent form (which is also shown in Fig. 5). This can be explained based on the solution (50), i.e. $\phi_{\text {Complete }} \equiv \phi_{\text {Main }}+\phi_{\text {Tail }}$, where $\phi_{\text {Main }}$ can be understood as the soliton structure obtained from the usual $\mathrm{KdV}$ equation in the case of homogeneous plasma and $\phi_{\text {Tail }}$ as the tailing structure (tanh profile) arising because of the plasma inhomogeneity due to the ionization. In the present case, the behavior depends completely on the variation of inhomgeneity constant $\mathrm{N}$ that appears in the solution through the constant $\mathrm{A}_{1}$. From the expression of $\mathrm{N}$, we can see that the inhomogeneity in the plasma is directly related to the ionization and hence, the amplification and damping of the tailing structure also depend. Thus, one can see that the ionization plays a vital role in determining the characteristic behavior of ion-acoustic solitons in spatially inhomogeneous plasmas [30]. An interesting result of the solitary wave solution in such plasmas is a continuous generation of the energy from the main soliton to the tailing structure. Thus, the tailing profile has a tendency to grow up as the soliton propagates in the inhomogeneous medium. The whole process of evolution of the tailing like structure depends on the order of the nonlinear and dispersive effects as compared to that of the inhomogeneity and ionization. These features exhibit only when the factor $\mathrm{N}$ appears in the $\mathrm{mKdV}$ equation. Thus, the combined contribution of inhomogeneity and ionization gives rise to the formation of a precursor in the soliton dynamics. The amplitude and width of the solitons depend on the generation of tailing structure that varies when the soliton propagates in the inhomogenous plasma. These results are very similar to the experimental observations, where the fission or production of a precursor behind the main soliton was realized [31].

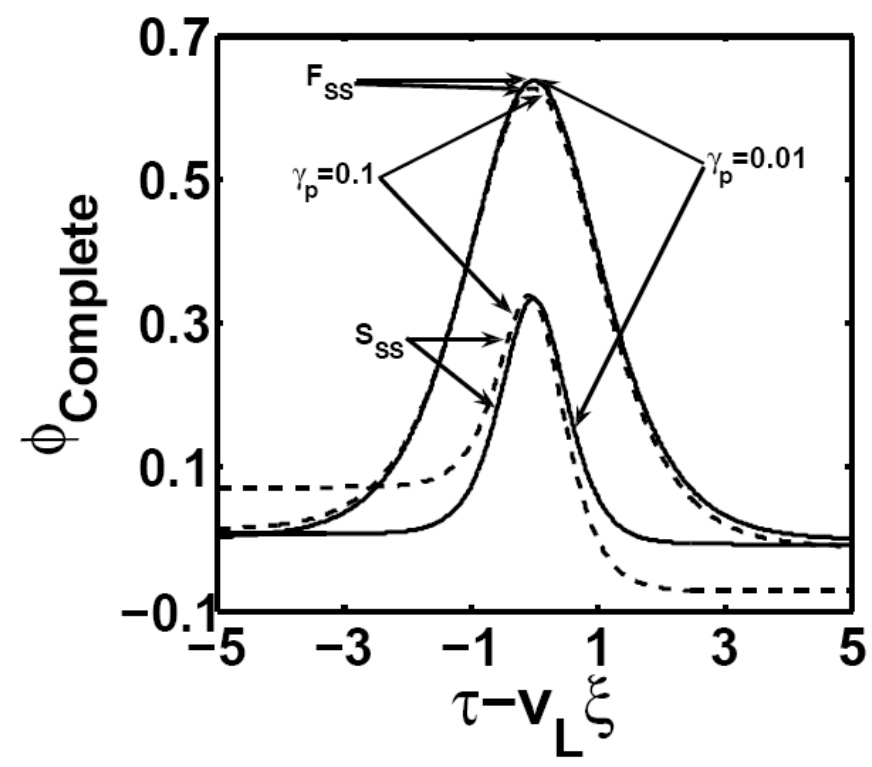

Fig. (4). Fast solitary structure $\mathrm{F}_{\mathrm{ss}}$ and slow solitary structure $\mathrm{S}_{\mathrm{ss}}$ for two different values of ionization rate $\gamma_{p}$, when $\mathrm{A}=0.5, \mathrm{n}_{\mathrm{p} 0}=0.9$, $\mathrm{Z}_{\mathrm{p}}=1, \theta=50^{\circ}, \sigma_{p}=0.01, v_{x 0}=v_{z 0}=0.08$ for fast mode, and $v_{x 0}=v_{z 0}=0.8$ for slow mode.

Fig. (6) shows the fast and slow solitary profiles for different values of the charge number, where larger widths of both the fast and slow solitons are observed for the higher value of $Z_{p}$. However, the amplitudes of these solitons behave oppositely and they show very weak dependence on $Z_{p}$. This is related to the weak variation of the phase velocity of both types of the modes with $Z_{p}$. On the other hand, Fig. (7) shows the fast and slow solitary structures for 


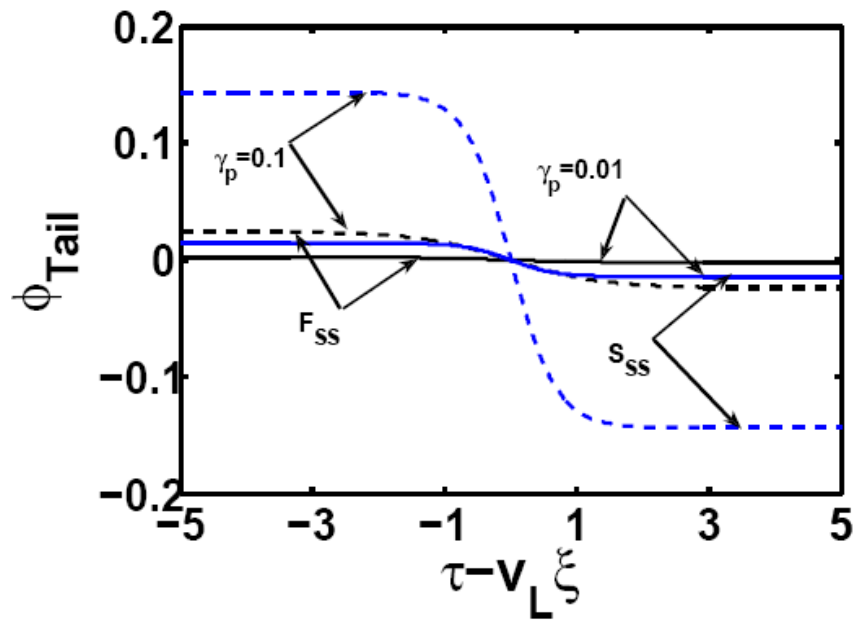

Fig. (5). Tailing structure $\varphi_{\text {Tail }} \equiv \phi_{\text {Tail }}$ of the fast and slow solitons, represented respectively by $\mathrm{F}_{\mathrm{ss}}$ and $\mathrm{S}_{\mathrm{ss}}$, for two different values of ionization rate $\gamma_{p}$, when $\mathrm{A}=0.5, \mathrm{n}_{\mathrm{p} 0}=0.9, \mathrm{Z}_{\mathrm{p}}=1, \theta=50^{\circ}$, $\sigma_{p}=0.01, v_{x 0}=v_{z 0}=0.08$ for fast mode, and $v_{x 0}=v_{z 0}=0.8$ for slow mode.

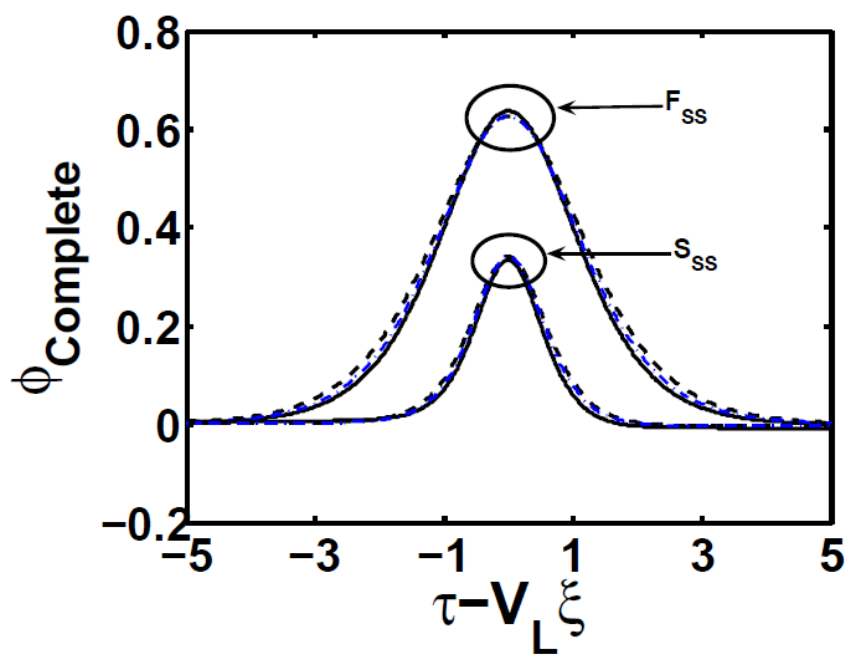

Fig. (6). Fast solitary structure $\left(\mathrm{F}_{\mathrm{ss}}\right)$ and slow solitary structure $\left(\mathrm{S}_{\mathrm{ss}}\right)$ for different values of $\mathrm{Z}_{\mathrm{p}}$, when $\mathrm{A}=0.5, \mathrm{n}_{\mathrm{p} 0}=0.9, \theta=50^{\circ}$, $\sigma_{p}=0.01, \gamma_{p}=0.01, v_{x 0}=v_{z 0}=0.08$ for fast mode, and $v_{x 0}=v_{z 0}=0.8$ for slow mode. Here solid lines are for $Z_{p}=1$ and dashed-dotted lines (middle ones) are for $Z_{p}=2$ and dashed lines (upper ones) are for $Z_{p}=3$ in an Ar gas plasma.

different values of ion to electron temperature ratio $\sigma_{p}$. Here, we see that the width decreases with the increase in $\sigma_{p}$ for both the fast and slow solitary structures. This is consistent with the observation made by Aziz and Stroth [14]. The amplitude of both the solitary structures is also reduced for higher $\sigma_{p}$, which is also seen by Malik and Singh [25] in a weakly relativistic magnetized plasma without ionization. This is very interesting to observe that the ion temperature, which was neglected by other investigators, modifies the solitary structures in a very significant manner. Also, the ion temperature has a pronounced effect on $\theta_{\text {min }}$ (Fig. 2). A comparison of Eq. (27) with Eq. (31) of [34] shows that the finite temperature limits the range of $\theta$ for the propagation of slow mode and hence, the slow solitary structure.

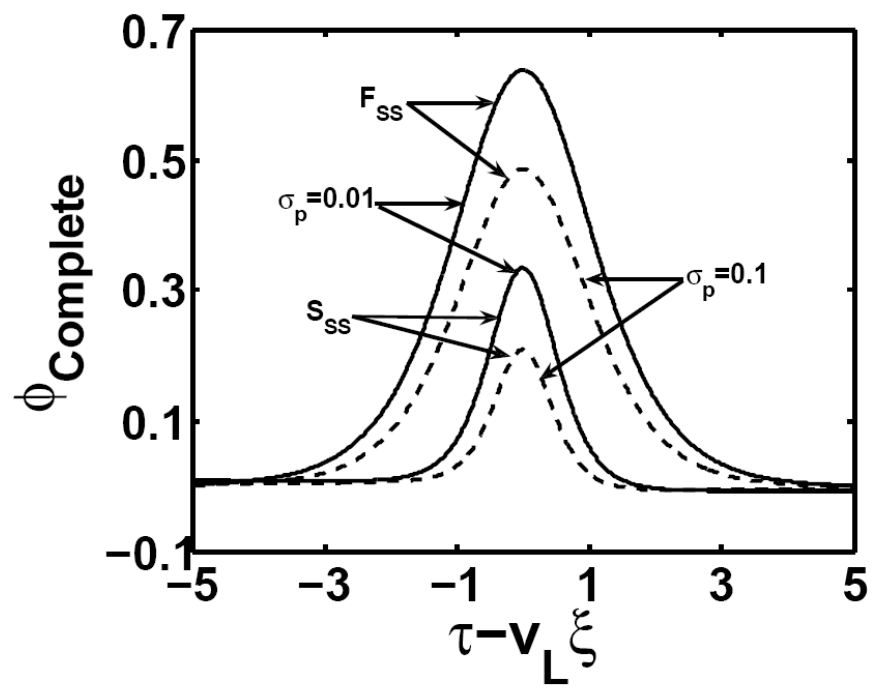

Fig. (7). Fast and slow solitary structures $\left(\varphi_{\text {Complete }}\right)$ for two different values of ion to electron temperature ratio, when $\mathrm{A}=0.5$, $\mathrm{n}_{\mathrm{p} 0}=0.9, \mathrm{Z}_{\mathrm{p}}=1, \theta=50^{\circ}, \gamma_{p}=0.01, v_{x 0}=v_{z 0}=0.08$ for fast mode, and $v_{x 0}=v_{z 0}=0.8$ for slow mode. Here, $\mathrm{F}_{\mathrm{ss}}\left(\mathrm{S}_{\mathrm{ss}}\right)$ represents the fast (slow) solitary structure.

\section{RECOMBINATION VERSUS IONIZATION}

So far we have considered the effect of ionization on the ion acoustic waves and their evolution as solitary structures. However, in real situation, when there are no external sources to cause the ionization, the recombination takes place due to internal collisions. The recombination may be Radiative Recombination (RR), collisional or Dielectronic Recombination (DR) or the Three Body Recombination (TBR) depending on the plasma characteristics, such as particle density, charge valency, temperature, etc. [56, 57]. Depending on the radiations emitted in the recombination processes (whether continuous or discrete frequency), we can estimate whether these are radiative recombination or dielectronic recombination. There are several processes like excitation (in the case of after-glow) that can also take place along with ionization and recombination. In our calculations, we neglect such excitation processes and consider the recombination process, which is the inverse of the ionization process taken into account in section IV. Hence, we assume the constant rate of the recombination and investigate the behavior of the fast and slow solitary structures based on the appropriate $\mathrm{mKdV}$ equation. The relevant $\mathrm{mKdV}$ equation is obtained, when $\gamma_{p}$ is taken as sink in the continuity equation, i.e. Eq. (5) is written as

$\frac{\partial n_{p}}{\partial t}+\frac{\partial\left(n_{p} v_{x}\right)}{\partial x}+\frac{\partial\left(n_{p} v_{z}\right)}{\partial z}+\gamma_{p}=0$.

Rest of the basic fluid equations remain the same. Following the same procedure, as used in section 3, we derive the $\mathrm{mKdV}$ equation in the case of recombination. This is given by 
$\frac{\partial \varphi_{1}}{\partial \tau}+K_{R} \varphi_{1} \frac{\partial \varphi_{1}}{\partial \xi}+L_{R} \frac{\partial^{3} \varphi_{1}}{\partial \xi^{3}}+M_{R} \varphi_{1} \frac{\partial n_{p 0}}{\partial \tau}+N_{R} \varphi_{1}=0$.

Here $K_{R}, L_{R}$ and $M_{R}$ are the same as $K, L$ and $M$ of Eq. (32), while $N_{R}$ is given by

$N_{R}=Q_{1 R}\left(\frac{-R R_{\lambda}}{2 Z_{p} \cos \theta n_{p 0}\left(R^{2} \lambda_{0}^{2}-\sigma_{p} \cos ^{2} \theta\right)-2 \sigma_{p} n_{p 0} \lambda_{0} R R_{\lambda}}\right)$,

together with

$Q_{1 R}=\frac{-2 \sigma_{p} Z_{p} \lambda_{0} \cos ^{2} \theta}{R_{\lambda}}-\frac{Z_{p} \lambda_{0} \cos \theta \gamma_{p} v_{z o}}{R_{\lambda}}$

$+\frac{2 Z_{p} \lambda_{0}^{2} R \gamma_{p}}{R_{\lambda}}+\frac{Z_{p} \lambda_{0}^{2} R \gamma_{p}}{R_{\lambda}}\left(-S \sin \theta \cos \theta+\cos ^{2} \theta\right)$

$S=\frac{\left(v_{z 0} \sin \theta-v_{x 0} \cos \theta\right)}{\left(v_{x o} \sin \theta+v_{z 0} \cos \theta\right)}$, and $R_{\lambda}=\frac{\lambda_{0}^{2} R^{2}-2 \sigma_{p} \cos ^{2} \theta}{\lambda_{0}^{2} R}$.

Eq. (51) is the similar type of $\mathrm{mKdV}$ equation as obtained in section 3. However, only the coefficient $\mathrm{N}$ is different in Eq. (51). In view of this, we directly use soliton solution (49) for investigating the soliton behavior in the case of recombination.

Fig. (8) shows the effect of the magnetic field on both the solitary structures corresponding to the fast and slow modes in a plasma, where the recombination is dominating. The effect of the magnetic field in the case of ionization has already been discussed in Fig. (3). When we compare Fig. (8) with Fig. (3), we find that in the case of recombination the tail is very much dominating as compared to the case of ionization. On the other hand, the effect of the recombination rate is shown in Fig. (9), where it is realized that the recombination process modifies the solitary structure in a very significant manner. It is interesting to note that the

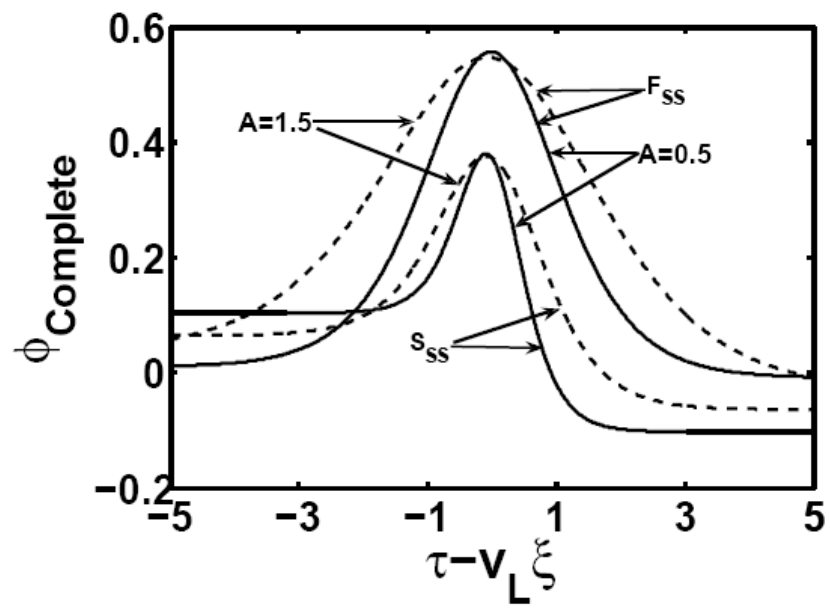

Fig. (8). Fast and slow solitary structures $\left(\varphi_{\text {Complete }}\right)$, showing the effect of magnetic field (through coefficient A), when $\mathrm{n}_{\mathrm{p} 0}=0.9$, $\gamma_{\mathrm{p}}=0.01, \mathrm{Z}_{\mathrm{p}}=1, \theta=50^{\circ}$ and $\sigma_{p}=0.01, v_{x 0}=v_{z 0}=0.08$ for fast mode, and $v_{x 0}=v_{z 0}=0.8$ for slow mode. $\mathrm{F}_{\mathrm{ss}}\left(\mathrm{S}_{\mathrm{ss}}\right)$ shows the fast (slow) solitary structure. structure corresponding to the slow mode is very drastically modified in comparison with the fast solitary structure. Actually this happens due to the coefficient $\mathrm{N}_{\mathrm{R}}$ that shows a strong dependence on the recombination rate. Since the growth of tailing structure is coupled with the energy exchange mechanism between the soliton and its tail, it is understood that the energy of the slow soliton is transferred at a higher rate to the tail, when the higher recombination takes place in the plasma. Hence, the tail grows very significantly in the case of fast recombination.

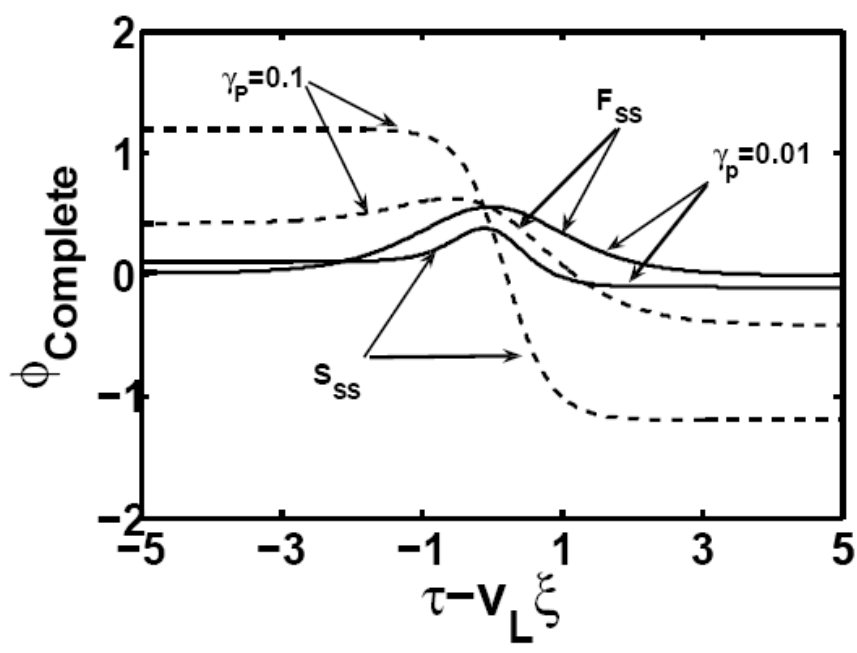

Fig. (9). Fast and slow solitary structures, showing the effect of recombination rate $\gamma_{p}$, when $\mathrm{A}=0.5, \mathrm{n}_{\mathrm{p} 0}=0.9, \mathrm{Z}_{\mathrm{p}}=1, \theta=50^{\circ}$, $\sigma_{p}=0.01, v_{x 0}=v_{z 0}=0.08$ for fast mode, and $v_{x 0}=v_{z 0}=0.8$ for slow mode.

In Fig. (10), we have examined the effect of ion temperature (which was neglected in earlier investigations) on the fast and slow solitary structures in the presence of the recombination. Here also, the similar effect of the ion temperature on the soliton amplitude is realized, as in the case of ionization. However, the point of observation is that the tailing structure shows a strong dependence on the ion temperature. In the case of only ionization the shape of the soliton structure remains almost the same, whereas in the case of only recombination the tailing structure grows very well and modifies the main soliton. For example, in the case of fast solitary structure the tail grows faster, when the ions carry higher temperature in the plasma. The slow solitary structure, however, shows opposite behavior and its tail becomes less prominent. Further, based on the magnitude of tails of the fast and slow solitary structures, it can be deduced that the transfer of energy between the soliton and its tailing structure is more in the case of slow solitary structure. This is also consistent to the observation made in Fig. (9).

In order to compare the solitary structures under the effects of ionization and recombination, when the ions carry more charge, we have plotted the effect of $Z_{p}$ on $\varphi_{\text {Complete }}$ in Fig. (11). On comparing this figure with Fig. (6), it is observed that the effect of the charge of ion is very weak on both the solitary structures in both the cases of ionization and recombination. However, the solitary structures with smaller amplitudes and larger widths are observed in the case of only recombination. 


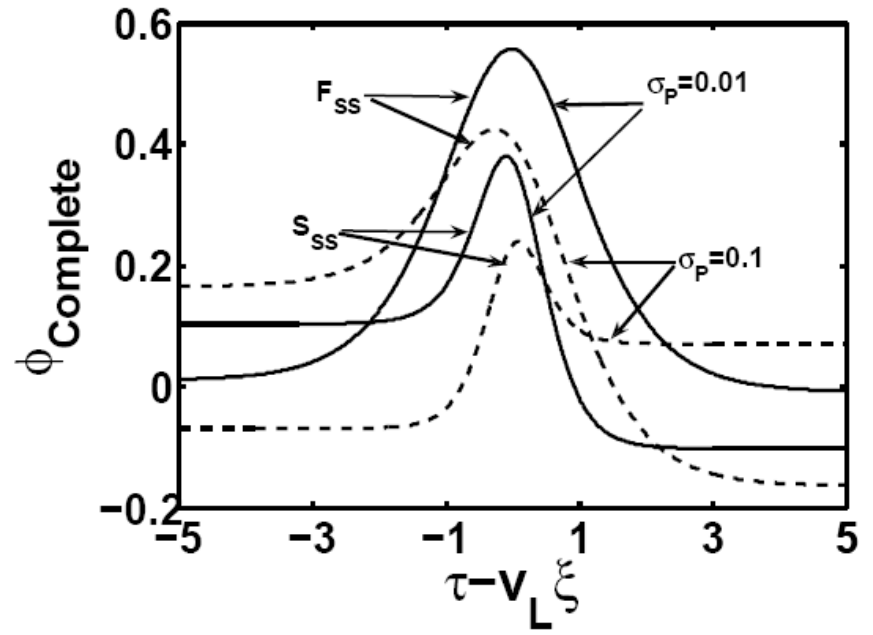

Fig. (10). Fast and slow solitary structures, showing the effect of ion temperature (ratio $\sigma_{p}$ ) in the case of recombination, when $\mathrm{n}_{\mathrm{p} 0}=0.9, \gamma_{\mathrm{p}}=0.01, \mathrm{Z}_{\mathrm{p}}=1, \theta=50^{\circ}, \mathrm{A}=0.5, v_{x 0}=v_{z 0}=0.08$ for fast mode, and $v_{x 0}=v_{z 0}=0.8$ for slow mode. $\mathrm{F}_{\mathrm{ss}}\left(\mathrm{S}_{\mathrm{ss}}\right)$ shows the fast (slow) solitary structure.

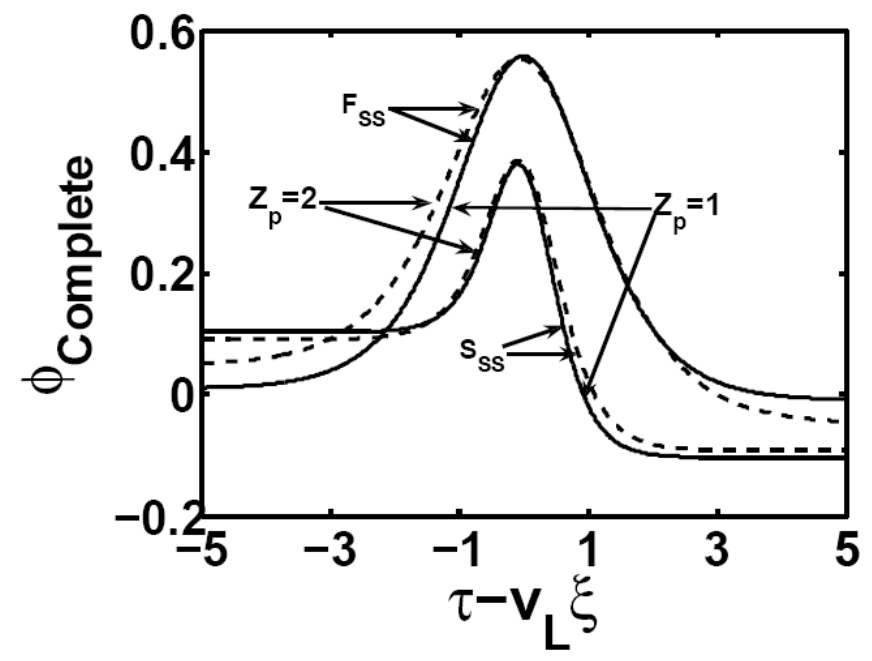

Fig. (11). Weak effect of charge valency $Z_{p}$ on the fast and slow solitary structures in the case of recombination, when $\mathrm{n}_{\mathrm{p} 0}=0.9$, $\gamma_{\mathrm{p}}=0.01, \sigma_{p}=0.01, \theta=50^{\circ}, \mathrm{A}=0.5, v_{x 0}=v_{z 0}=0.08$ for fast mode, and $v_{x 0}=v_{z 0}=0.8$ for slow mode. $\mathrm{F}_{\mathrm{ss}}\left(\mathrm{S}_{\mathrm{ss}}\right)$ shows the fast (slow) solitary structure.

In order to see the effects of the density gradient on the solitary structures in the cases of ionization and recombination, we have plotted $\varphi_{\text {Complete }}$ for different values of $n_{p 0 \tau}$ in Figs. $(12,13)$, respectively. On comparison, we find that the soliton maximum increases with increasing $n_{p 0 \tau}$ for the slow solitary structure as well as for the fast solitary structure, when we consider the ionization in the plasma (Fig. 12); the same is true for the case of the recombination also (Fig. 13). However, the effect of the density gradient is most significant on the tailing structure in the case of recombination. Moreover, we find that the soliton amplitude is enhanced by $\sim 14 \%$ in the case of ionization, whereas it is increased by $\sim 11 \%$ when the recombination takes place. Over and all, we conclude that the density gradient modifies the solitary structures very significantly in the case of recombination.

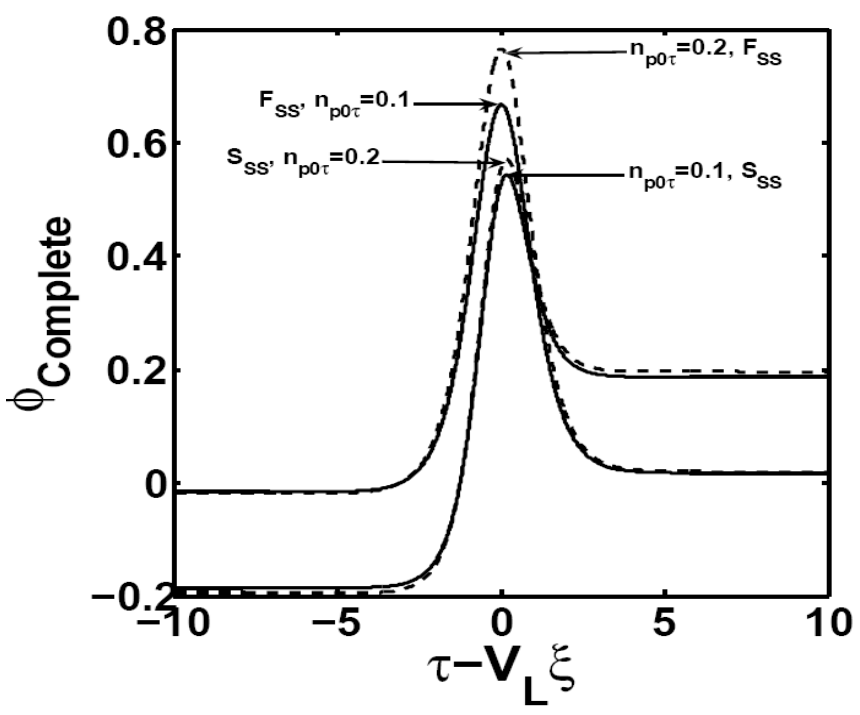

Fig. (12). Variation of soliton profile with change in density gradient for the case of ionization when $\mathrm{n}_{\mathrm{p} 0}=0.9, \gamma_{\mathrm{p}}=0.01$, $\sigma_{p}=0.01, \mathrm{Z}_{\mathrm{p}}=1, \theta=55^{\circ}, \mathrm{A}=0.5, v_{x 0}=v_{z 0}=0.09$ for fast mode, and $\theta=50^{\circ}, v_{x 0}=v_{z 0}=0.9$ for slow mode. $\mathrm{F}_{\mathrm{ss}}\left(\mathrm{S}_{\mathrm{ss}}\right)$ shows the fast (slow) solitary structure.

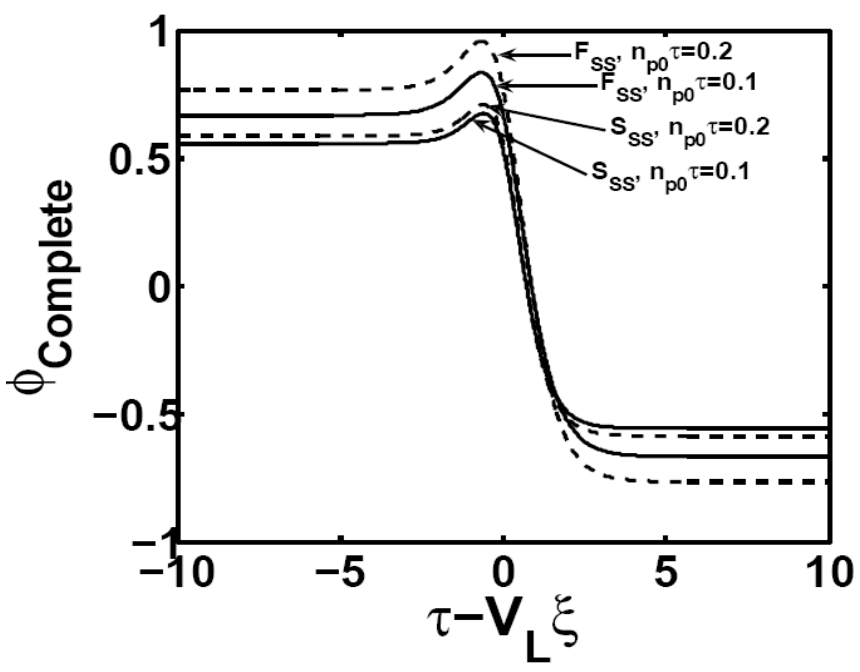

Fig. (13). Variation of soliton profile with change in density gradient for the case of recombination, when $\mathrm{n}_{\mathrm{p} 0}=0.9, \gamma_{\mathrm{p}}=0.01$, $\sigma_{p}=0.01, \theta=55^{\circ}, \mathrm{Z}_{\mathrm{p}}=1, \mathrm{~A}=0.5, v_{x 0}=v_{z 0}=0.09$ for fast mode, and $\theta=50^{\circ}, v_{x 0}=v_{z 0}=0.9$ for slow mode. $\mathrm{F}_{\mathrm{ss}}\left(\mathrm{S}_{\mathrm{ss}}\right)$ shows the fast (slow) solitary structure.

We can find the set of plasma parameters for which the tailing structure associated with the soliton is disappeared for a particular/critical value of the ionization and recombination. Since in the case of ionization the coefficient $\mathrm{N}$ encompasses the contribution of the ionization rate, we can find the critical value of the ionization rate by setting this coefficient to zero so that the soliton solution does not carry the coefficient $\mathrm{N}$ and hence, the effect of ionization. The critical value of the ionization rate constant for which the tailing structure will disappear is given by the following expression: 
$\gamma_{p c}=\frac{-2 \sigma_{p} \cos \theta\left(v_{\theta}\right)}{v_{z 0}\left\{v_{\theta} \pm \sqrt{Z_{p}+2 \sigma_{p}} \cos \theta\right\}}$.

Here $v_{\theta}$ is the same as defined earlier for Eq. (32). Here, negative (positive) sign in the denominator corresponds to the fast (slow) solitary structure. From this expression we can see that $\gamma_{p c}$ will be positive only when $v_{x 0} \sin \theta+v_{z 0} \cos \theta<\sqrt{Z_{p}+2 \sigma_{p}} \cos \theta$. This provides an upper limit on the angle $\theta$, as given below

$\theta_{\max }=\tan ^{-1}\left(\frac{\sqrt{Z_{p}+2 \sigma_{p}}-v_{z 0}}{v_{x 0}}\right)$.

Our numerical calculations show that $\gamma_{p c}$ will always be negative for the case of slow solitary structure. Hence, the coefficient $\mathrm{N}$ will not vanish in the case of slow structure and the tailing structure will always be associated with the slow soliton. However, the tail will disappear for the fast solitary structure, if the ionization rate matches the value of $\gamma_{p c}$ for the given set of parameters. On the other hand, for the case of recombination, the critical value of the recombination rate constant is obtained by setting the coefficient $\mathrm{N}_{\mathrm{R}}$ to zero. This is given by

$$
\begin{aligned}
\gamma_{p c}= & \frac{2 \sigma_{p} v_{\theta} \cos \theta}{v_{z 0}\left(-v_{\theta} \pm \sqrt{Z_{p}+2 \sigma_{p}} \cos \theta \cos 2 \theta\right)} . \\
& \pm 2 \sqrt{Z_{p}+2 \sigma_{p}}\left(v_{x 0} \cos ^{2} \theta \sin \theta+v_{\theta}\right)
\end{aligned}
$$

Here, negative (positive) sign in the denominator corresponds to the fast (slow) solitary structure. It can be obtained from Eq. (56) that for the case of recombination $\gamma_{p c}$ will be positive only for the slow solitary structure. However, this is possible only when the following condition is satisfied

$v_{z 0}+\frac{2 \sqrt{Z_{p}+2 \sigma_{p}}\left(v_{\theta}+\cos ^{2} \theta \sin \theta v_{x 0}\right)}{\sqrt{Z_{p}+2 \sigma_{p}} \cos \theta \cos 2 \theta-v_{\theta}}>0$.

Hence, we can say that the tailing structure will disappear in the case of the slow solitary structure, when the recombination effect is dominating, while a tail will always accompany the main soliton for the fast solitary structure. On the other hand, the tailing structure will disappear in the case of the fast solitary structure, when the ionization effect is dominating, while a tail will always accompany the main soliton for the slow solitary structure. These solitary structures without tail are shown in Fig. (14) for the critical values of the ionization and recombination for the fast and slow solitary structures, respectively.

Fig. (15) shows the dependence of the critical values of the ionization and the recombination rate constants $\gamma_{p c}$ on $\sigma_{p}, \theta, v_{x 0}$ and $v_{z 0}$. Here, it is evident that $\gamma_{p c}$ for both the cases of the ionization (solid lines) and recombination (dashed lines) attain larger values for the higher values of $\sigma_{p}$. It means, if the plasma contains higher temperature ions, a faster rate of the ionization/recombination rate is required so that the solitary structures do not see the effect of the ionization/recombination; similar is the case for the plasma where the ions with higher drifts are present. However, the effect of angle $\theta$ is opposite in the cases of ionization and recombination. Higher value of $\gamma_{p c}$ is required in the case of the ionization, if the magnetic field is applied at a larger angle $\theta$. However, lower value of $\gamma_{p c}$ is required in the case of the recombination, if the magnetic field is applied at a larger angle $\theta$.

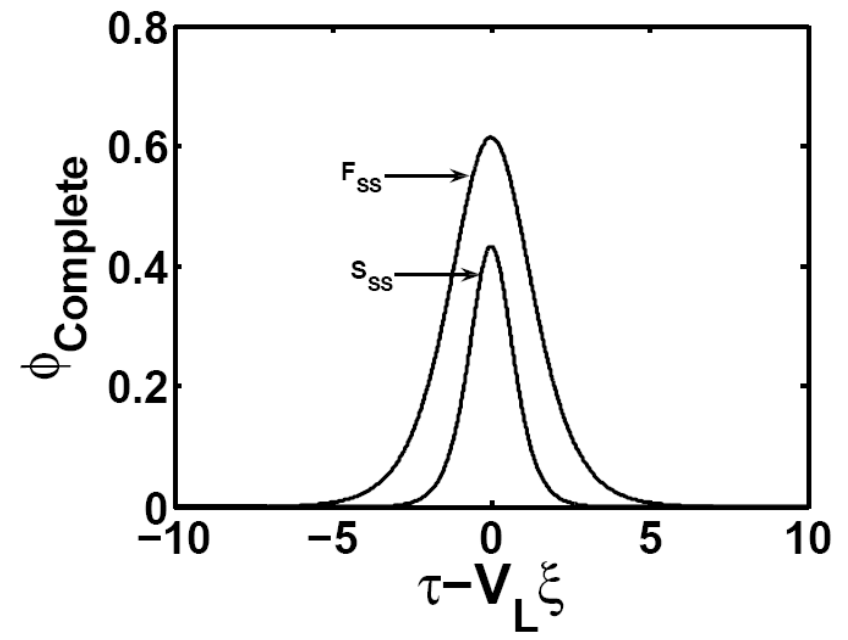

Fig. (14). Complete soliton for the critical values of ionization and recombination rates $\gamma_{p c}$, when $\mathrm{n}_{\mathrm{p} 0}=0.9, \sigma_{p}=0.01, \theta=55^{\circ}$, $\mathrm{Z}_{\mathrm{p}}=1, \mathrm{~A}=0.5, v_{x 0}=v_{z 0}=0.09$ for fast structure (Fss), and $\theta=$ $50^{\circ} v_{x 0}=v_{z 0}=0.9$ for slow solitary structure $\left(\mathrm{S}_{\mathrm{ss}}\right)$.

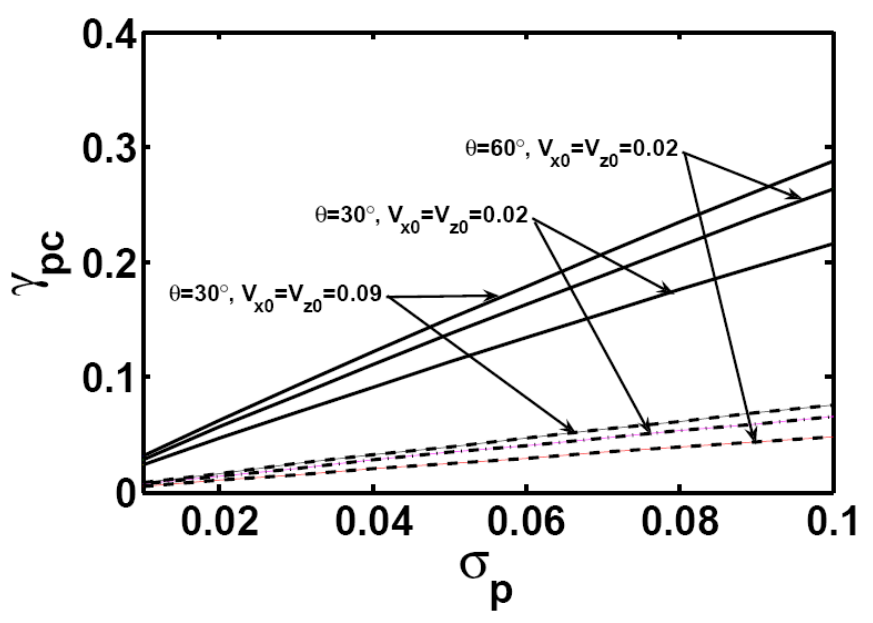

Fig. (15). Variation of critical ionization (recombination) rate $\gamma_{p c}$ constant with ion to electron temperature ratio $\sigma_{p}$ for different values of $\theta$ and drift velocities. Here solid (dashed) lines represent the case of ionization (recombination) for singly charged ions $\left(\mathrm{Z}_{\mathrm{p}}=1\right)$.

\section{ESTIMATION OF IONIZATION/RECOMBINATION EFFECT}

With regard to the estimation of the ionization/recombination effects on the soliton propagation for typical plasma 
parameters of laboratory and space, and the observation of ionization/recombination-modified solitary structures in the experiments, we discuss the ratio of amplitudes of the tail and the main soliton. For a typical laboratory plasma, kept in a chamber of $\sim 40 \mathrm{~cm}$ diameter and when plasma density $n_{00}$ $=10^{14} / \mathrm{m}^{3}, \mathrm{~B}_{0}=0.1 \mathrm{~T}, \mathrm{~T}_{\mathrm{e}}=3 \mathrm{eV}$ and $\mathrm{T}_{\mathrm{i}}=0.3 \mathrm{eV}$, we find that the Larmor radius $\sim 5 \times 10^{4} \mathrm{~m}$ (considering the thermal velocity as perpendicular component of velocity $v_{\perp}$ ) and the tail is $\sim 7 \%$ of soliton amplitude that is enhanced to $\sim 23 \%$, when the ion drift is reduced from $0.6 \mathrm{C}_{\mathrm{s}}$ to $0.06 \mathrm{C}_{\mathrm{s}}\left(\mathrm{C}_{\mathrm{s}}\right.$ is the ion acoustic speed) in the hydrogen plasma. However, the tail is $27 \%$ of soliton amplitude that is enhanced to $\sim 78 \%$, when the ion drift is reduced from $0.6 \mathrm{C}_{\mathrm{s}}$ to $0.06 \mathrm{C}_{\mathrm{s}}$ in the argon plasma. The tailing structure is found to reduce if the magnetic field is increased or the plasma density is enhanced. Hence, this is clear that these modified solitary structures can be easily observed in the laboratory. In view of space plasma, we can take the example of Van Allen radiation belts, which are dominated by the protons and have the parameters: $n_{00} \sim 10^{8} / \mathrm{m}^{3}, \mathrm{~T}_{\mathrm{i}}=1 \mathrm{eV}, \mathrm{T}_{\mathrm{e}} \sim 1000 \mathrm{eV}$ and $\mathrm{B}=5 \times 10^{7} \mathrm{~T}$. Here, the Larmor radius comes out to be $\sim 200 \mathrm{~m}$ and the tail is $\sim 42 \%$, when the protons drift with the speed of $0.6 \mathrm{C}_{\mathrm{s}}$. However, the tail is $\sim 89 \%$, when the protons drift with the speed of $0.06 \mathrm{C}_{\mathrm{s}}$. Hence, the solitary structures with prominent tail can be observed in the space related plasma, where the plasma density and the magnetic field are smaller in comparison with the laboratory plasmas.

\section{CONCLUDING REMARKS}

The present calculations describe the real situation of the plasma, where the ionization and/or recombination take place and the ions carry finite temperature. A modified form of the $\mathrm{KdV}$ equation governs the behavior of solitary waves in this type of plasma. We have discussed separately the cases of ionization and recombination, and compared the two for better understanding of occurrence of solitary structures. In both the cases, the ion temperature is found to reduce the amplitude significantly. Consistent to the investigations made by other investigators, the dispersive property of the plasma is found to be modified in the presence of magnetic field, but the solitons with wider widths evolve under the effect of stronger magnetic field in a plasma having ionization or recombination. The main soliton is found to propagate along with its tailing structure, when the ionization or recombination takes place in the plasma, and the tail with more prominent form arises in the case of recombination; the same is observed in the case of stronger density gradient. The effect of charge of the ions affects only the tailing structure; however, the tail shows weak dependence on the charge number. We have also derived the expressions for critical rates of ionization and recombination, based on which it is deduced that the fast solitary structure in the case of ionization and the slow solitary structure in the case of recombination can evolve without their tailing structures.

\section{CONFLICT OF INTEREST}

The authors confirm that this article content has no conflicts of interest.

\section{ACKNOWLEDGEMENTS}

The Council of Scientific and Industrial Research (CSIR), Govt. of India is gratefully acknowledged for rendering the financial assistance for carrying out this work.

\section{REFERENCES}

[1] Washimi H, Taniuti T. Propagation of ion-acoustic solitary waves of small amplitude. Phys Rev Lett 1966; 17: 996-8.

[2] Gardner CS, Greene JM, Kruskal MD. Method for solving the Korteveg-deVries equation. Phys Rev Lett 1967; 19: 1095-7.

[3] Taylor RJ, Baker DR, Ikezi H. Observation of collisionless electrostatic shocks. Phys Rev Lett 1970; 24: 206-9.

[4] Rao NN, Verma RK. Modified Korteweg-deVries equation for spatially inhomogeneous plasmas. Phys Lett A 1979; 70 A: 9-11.

[5] Ko K, Kuehl HH. Korteweg-deVries soliton in a slowly varying medium. Phys Rev Lett 1978; 40: 233-6.

[6] Nishikawa K, Kaw PK. Propagation of solitary ion-acoustic waves in inhomogeneous plasmas. Phys Lett A 1975; 50: 455-6.

[7] Nishida Y. Reflection of a planar ion-acoustic soliton from a finite plane boundary. Phys Fluids 1984; 27: 2176-80.

[8] Nishida Y, Nagasawa T. Excitation of ion-acoustic rarefactive solitons in a two-electron-temperature plasma. Phys Fluids 1986; 29: 345-8.

[9] Kuehl HH. Reflection of ion-acoustic soliton by plasma inhomogeneities. Phys Fluids 1983; 26: 1577-83.

[10] Nagasawa T, Nishida Y. Non-linear reflection and refraction of a planar ion-acoustic plasma solitons. Phys Rev Lett 1986; 56: 268891.

[11] Kuehl HH, Imen K. Finite-amplitude ion-acoustic solitons in weakly inhomogeneous plasmas. Phys Fluids 1985; 28: 2375-81.

[12] Nejoh Y. The effect of the ion temperature on the ion acoustic solitary waves in a collissionless relativistic plasma. J Plasma Phys 1987; 37: 487-95.

[13] Singh S, Dahiya RP. Effect of ion temperature and plasma density on an ion-acoustic soliton in a collisionless relativistic plasma: an application to radiation belt. Phys Fluids B 1990; 2: 901-6.

[14] Aziz F, Stroth U. Effect of trapped electron on soliton propagation in a plasma having density gradient. Phys Plasmas 2009; 16: 032108 (1-7).

[15] Nakamura Y, Tsukabayashi I. Observation of modified KortewegdeVries solitons in a multicomponent plasma with negative ions. Phys Rev Lett 1984; 52: 2356-9.

[16] Nakamura Y, Tsukayabashi I, Ludwig GO, Ferreira JL. Large amplitude solitary waves in a multicomponent plasma with negative ions. Phys Lett A 1985; 113: 155-8.

[17] Nakamura Y. Experiments on ion acoustic solitons in a plasma. IEEE Trans Plasma Sci 1982; 10: 180-95.

[18] Chang HY, Raychaudhuri S, Hill J, Tsikis EK, Lonngren KE. Propagation of an ion-acoustic soliton in an inhomogeneous plasma. Phys Fluids 1986; 29: 294-7.

[19] Cooney JL, Aossey DW, Williams JE, Lonngren KE. Experiments on grid-excited solitons in positive-ion-negative-ion plasma. Phys Rev E 1993; 47: 564-9.

[20] Cooney JL, Gavin MT, Lonngren KE. Experiments on KortewegdeVries solitons in a positive-ion negative-ion plasma. Phys Fluids B 1991; 3: 2758-66.

[21] Yi S, Cooney JL, Kim H, Amin A, El-Zein Y, Lonngren KE. Reflection of modified Korteweg-deVries soliton in a negative ion plasma. Phys Plasmas 1996; 3: 529-35.

[22] Cooney JL, Gavin MT, Williams JE, Aossey DW, Lonngren KE. Soliton propagation, collision, and reflection at appositive ion negative ion plasma. Phys Fluids B 1991; 3: 3277-81.

[23] Mishra MK, Chhabra RS, Sharma SR. Obliquely propagating ionacoustic soliton in a multicomponent magnetized negative ion plasma. J Plasma Phys 1994; 52: 409-29.

[24] Singh DK, Malik HK. Modified Korteweg-deVries soliton evolution at a critical density of negative ions in an inhomogeneous magnetized cold plasma. Phys Plasmas 2007; 14: 062113 (1-8).

[25] Malik HK, Singh K. Small amplitude soliton propagation in a weakly relativistic magnetized space plasma: electron inertia contribution. IEEE Trans Plasma Sci 2005; 33: 1995-2004. 
[26] Singh K, Kumar V, Malik HK. Electron inertia contribution to soliton evolution in an inhomogeneous weakly relativistic two fluid plasma. Phys Plasmas 2005; 12: 072302 (1-8).

[27] Singh DK, Malik HK. Reflection of non-linear solitary waves (m$\mathrm{KdV}$ solitons) at critical density of negative ions in a magnetized plasma. Plasma Phys Control Fus 2007; 49: 1551-63.

[28] Malik HK. Ion acoustic solitons in a weakly relativistic magnetized warm plasma. Phys Rev E 1996; 54: 5844-7.

[29] El-Labany SK, El-Shamy EF. Critical density solitary waves structures in a hot magnetized dusty plasma with vortex-like ion distribution in a phase space. Phys Plasmas 2005; 12: 04230 (1-6).

[30] Shivamoggi BK. Effect of finite ion-temperature on ion-acoustic solitary waves in an inhomogeneous plasma. Can J Phys 1981; 59: 719-21.

[31] Watanable S, Yajima N. KdV soliton in inhomogeneous system. J Phys Soc Jpn 1984; 53: 3325-34.

[32] Malik HK, Soliton reflection in magnetized plasma: effect of ion temperature and non-isothermal electrons. Phys Plasmas 2008; 15: $072105(1-8)$.

[33] Singh DK, Malik HK. Soliton reflection in a negative ion containing plasma: effect of magnetic field and ion temperature. Phys Plasmas 2006; 13: 082104 (1-10).

[34] Jyoti, Malik HK. Solitary wave evolution in a magnetized plasma under the ionization effect. Phys Plasmas 2011; 18: 102116 (1-8)

[35] Kostrov A, Luchinin G, Permitin G, Starodubtsev M. Effect of spatial dispersion on the propagation of electromagnetic waves in a magnetoplasma in the upper-hybrid frequency range. The $29^{\text {th }}$ IEEE Int Conf Plasma Sci 2002; 301.

[36] Starodubtsev M, Al-Hassan MK, Ito H, Yugami N, Nishida Y. The $29^{\text {th }}$ IEEE Int Conf Plasma Sci 2002; 303.

[37] Malik HK. Ion acoustic solitons in a relativistic warm plasma with density gradient. IEEE Trans Plasma Sci 1995; 23: 813-5.

[38] Nejoh YN. The effect of trapped electrons on large amplitude ionacoustic waves in a plasma with an electron beam. IEEE Trans Plasma Sci 1996; 24: 1224-8.

[39] Lonngren KE. A two-dimensional soliton in a positive ionnegative ion plasma. IEEE Trans Plasma Sci 1991; 19: 1259-66.

[40] Nakamura Y. Experiments on ion-acoustic solitons in plasmas. IEEE Trans Plasma Sci 1982; 10: 180-95.
[41] Khazei M, Gabl EF, Bulson JM, Sheerin JP, Nakamura Y, Lonngren KE. Ion-acoustic soliton propagation in a density well. IEEE Trans Plasma Sci 1983; 11: 46-7.

[42] Dorranian D, Sabetkar A. Dust acoustic solitary waves in a dusty plasma with two kinds of nonthermal ions at different temperatures. Phys Plasmas 2012; 19: 13702 (1-6)

[43] Cobine JD. Gaseous Conductors. New York: Dover 1958

[44] Millman J, Seely S. Electronics (New York: McGraw-Hill) Chapters 9, 10 and $11 ; 1951$.

[45] Lehnert B. Ionization process of a plasma. Phys Fluids 1966; 9: 774-9.

[46] Engel AV, Steenbeck M. Edwards Broth Inc; Ann Arbor Michigan 1944.

[47] Shivamoggi BK. Ion-Acoustic solitary waves in an inhomogeneous plasma. J Plasma Phys 1988; 40: 579-83.

[48] Das GC, Sarma MK. Evolution of ion-acoustic solitary wave in an inhomogeneous discharge plasma. Phys Plasmas 2000; 7: 3964-9.

[49] Chauhan SS, Malik HK, Dahiya RP. Reflection of ion acoustic soliton having negative ions. Phys Plasmas 1996; 3: 3932-8.

[50] Singh S, Dahiya RP. Propagation characteristics and reflection of an ion-acoustic soliton in an inhomogeneous plasma having warm ions. J Plasma Phys 1989; 41: 185-97.

[51] Asano N, Taniuti T. Reductive perturbation method for nonlinear inhomogeneous media: II. J Phys Soc Jpn 1970; 29: 209-14.

[52] Asano N. Reductive perturbation method for nonlinear inhomogeneous media: III. J Phys Soc Jpn 1970; 29: 220-4.

[53] Das GC, Karmakar B, Singh KI. Solitons in a relativistic plasma with negative ions. IEEE Trans Plasma Sci 1990; 18: 86-90.

[54] Malik HK, Singh DK, Nishida Y. On reflection of solitary waves in magnetized multicomponent plasma with non-isothermal electrons. Phys Plasmas 2009; 16: 72112 (1-7).

[55] Yan C. A simple transformation for nonlinear waves. Phys Lett A 1996; 224: 77-84.

[56] Hahn Y. Electron-ion recombination - an overview. Rep Prog Phys 1997; 60: 691-59.

[57] Barbet A, Sadeghi N, Pebay-Preyroula JC. Study of electron-ion recombination processes in the xenon afterglow plasma. J Phys B: At Mol Phys 1975; 8: 1785-94. 